\title{
Recent Advances in Asthma Management
}

\author{
An Expert Interview with Peter J Barnes
}

National Heart and Lung Institute, Imperial College, London, UK

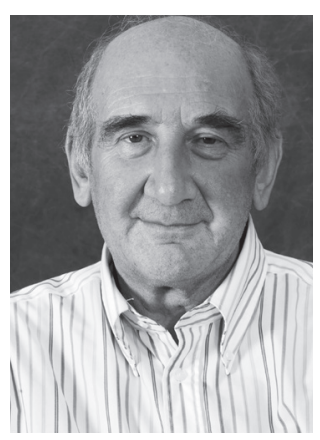

DOl: https://doi.org/10.17925/ERPD.2018.4.1.15

\section{Peter J Barnes}

Peter I Barnes is Margaret Turner-Warwick Professor of Medicine at the National Heart and Lung Institute, Head of Respiratory Medicine at Imperial College and Honorary Consultant Physician at Royal Brompton Hospital, London. He qualified at Cambridge and Oxford Universities (first class honours) and was appointed to his present post in 1987. Professor Barnes has published over 1,000 peer-reviewed papers on asthma, chronic obstructive pulmonary disorder and related topics (h-index 161) and has written or edited over 50 books. He is amongst the 20 most highly cited researchers in the world and has been the most highly cited respiratory researcher in the world over the last 20 years. He was elected a Fellow of the Royal Society in 2007, the first respiratory researcher for over 150 years. Professor Barnes has given several prestigious lectures, including the Amberson Lecture at the American Thoracic Society (ATS) and the Sadoul Lecture at the European Respiratory Society (ERS), and was President of the latter in 2013-4.

\section{Keywords}

Adherence, anti-interleukin-5, asthma, cytokines, eosinophils, interleukins, mild asthma, monoclonal antibodies, reliever, severe asthma

Disclosure: Peter J Barnes has received research funding from AstraZeneca and Boehringer Ingelheim and has been on advisory boards and given lectures for Astrazeneca, Boehringer Ingelheim, Chiesi, Novartis and Teva Pharmaceutical Industries Ltd.

Review Process: This is an expert interview and, as such, has not undergone the journal's standard peer review process.

Acknowledgement: Medical writing assistance was provided by Katrina Mountfort of Touch Medical Media and was supported by Touch Medical Media.

Authorship: The named author meets the International Committee of Medical Journal Editors (ICMJE) criteria for authorship of this manuscript, takes responsibility for the integrity of the work as a whole, and has given final approval for the version to be published.

open Access: This article is published under the Creative commons Attribution Non-commercial License, which permits any non-commercial use, distribution, adaptation, and reproduction provided the original author and source are given appropriate credit. () The Author 2018

Received: 11 July 2018

Published Online: 14 August 2018

Citation: European Respiratory \& Pulmonary Diseases. 2018;4(1):15-6

Corresponding Author: Peter J Barnes, National Heart and Lung Institute, Imperial College School of Medicine, Dovehouse Street, London SW3 6LY, UK. E: p.j.barnes@imperial.ac.uk

Support: No funding was received in the publication of this article.
$\mathrm{T}$ he treatment of asthma has improved greatly during the last two decades, and deaths from the disease have decreased. ${ }^{1}$ Despite these advances, many asthma patients fail to achieve optimal asthma control as defined by international guidelines. ${ }^{2,3}$ Severe asthma is associated with high risk of exacerbations and death. ${ }^{4}$ Definitions of severe asthma vary but, according to European Respiratory Society (ERS)/American Thoracic Society (ATS) and Global Initiative for Asthma (GINA) guidelines, severe asthma is asthma that requires treatment with high doses of inhaled corticosteroids plus a second controller, and/or systemic corticosteroids, to prevent it from becoming uncontrolled, or remains uncontrolled despite this therapy. ${ }^{2.5}$ In an expert interview, Professor Barnes discusses the latest advances in the management of both mild and severe asthma.

\section{Q. What are the limitations of current reliever medications in mild asthma?}

By far the most commonly used reliever medications in asthma are short-acting beta agonists (SABA) such as salbutamol and terbutaline. While these give rapid relief of symptoms, they don't reduce the underlying inflammation of asthma. In fact, there is evidence that overuse of SABA can make asthma control worse, may increase inflammation and may increase the risk of death from asthma. ${ }^{6}$

\section{Q. Can you tell us a little about the SYGMA programme and the significance of the findings?}

The SYGMA programme aimed to investigate budesonide/formoterol (Symbicort ${ }^{\circledR}$, AstraZeneca, Cambridge, UK) given as needed for the treatment of mild asthma. Although it is recommended in GINA that in mild asthma, GINA step 1 patients, i.e., those with mild episodic asthma, should be treated with SABA alone and GINA step 2 patients who have persistent mild asthma should be treated with low-dose inhaled steroids plus SABA. ${ }^{2}$ However, very few studies have looked at the most effective treatment for mild asthma; most clinical trials have been in moderate to severe asthma.

The SYGMA studies were designed to investigate the possibility of an anti-inflammatory reliever instead of a SABA and compared this with the current recommendations of either SABA for GINA step 1 patients and low-dose inhaled steroids plus SABA for step 2 patients. Two studies were conducted, both of which were large placebo-controlled clinical trials and were undertaken for a year. ${ }^{7.8}$ Around 4,000 patients were enrolled in each study. In SYGMA-1, three treatments were compared: SABA reliever alone, budesonide/formoterol reliever alone, and low-dose inhaled steroids plus SABA alone.? SYGMA-1 clearly showed that the budesonide/formoterol reliever was more effective in controlling asthma than the SABA reliever and also reduced exacerbations 
significantly. The low-dose inhaled steroids plus SABA treatment was equivalent to the budesonide/formoterol reliever in terms of asthma control and reducing asthma exacerbation, however, in this trial, adherence to inhaled steroid maintenance was extremely high (around $80 \%$ ) because patients were reminded to take their medication and also knew that they were being monitored with electronic recording of their medication use. By contrast, real-world adherence is less than $20 \%$. This would strongly suggest that the budesonide/formoterol reliever should be the treatment of choice in the future. In addition, the dose of inhaled steroid that was needed in the budesonide/formoterol reliever group was about $20 \%$ of that of regular inhaled steroid. ${ }^{\text {? }}$

The second SYGMA study had two groups. The first was the budesonide/formoterol reliever, the second was low-dose inhaled steroids plus SABA reliever. ${ }^{8}$ These two treatments were found to be equivalent in terms of controlling asthma and reducing exacerbations but budesonide/formoterol achieved this control at around $25 \%$ of the regular inhaled steroid dose, confirming that the anti-inflammatory reliever is the treatment of choice for these patients. ${ }^{8}$

\section{Q. How has our increased understanding of endotypes informed our treatment of severe asthma?}

It is now recognised that severe asthma may have several subtypes. These can be differentiated by looking at inflammatory cells in the sputum. Around half of people with severe asthma have increased eosinophils despite the fact that they are taking maximum doses of inhaled steroids. The other inflammatory phenotypes are neutrophilic, where there is a predominance of neutrophils or a mixed pattern with eosinophils and neutrophils. The third phenotype is called paucigranulocytic, where there is no increase of inflammatory cells despite the patient experiencing symptoms. This has helped target asthma treatments towards eosinophilic inflammation.

\section{Q. What is the efficacy and safety of humanised monoclonal antibodies against interleukin-5 in severe eosinophilic asthma?}

In early studies of anti-interleukin-5 (IL-5) treatment, there was no effect overall in improving asthma control or reducing asthma exacerbations. ${ }^{9}$ However, subsequent studies selected patients with severe eosinophilic asthma as detected by sputum eosinophils, ${ }^{10-14}$ although we now know that blood eosinophils are equally effective at detecting eosinophilic asthma. These patients responded well to
anti-IL-5 treatment in terms of reduced exacerbations. In addition, it was possible to reduce the dose of oral steroids in patients with very severe asthma who were on maintenance oral steroids. The treatment didn't improve symptoms of lung function or quality of life, so it is not an alternative to inhaled steroids, but it seems to be a useful add-on treatment in patients with severe and very severe asthma who have high eosinophil levels.

\section{Q. What have been the most important recent developments in other monoclonal antibodies targeting different cytokines such as IL-13, IL-4, IL-17 and thymic stromal lymphopoietin?}

Several studies have reported the effects of these antibodies on asthma. Anti-IL-13 antibodies proved not to be very effective in improving asthma control or reducing exacerbations, but an antibody that blocks the common receptor for IL-4 and IL-13, IL-4 receptor alpha, has proved to be very effective. This treatment not only reduces exacerbations but improves lung function and symptoms, as well as quality of life. This treatment, which is called dupilumab (Dupixent ${ }^{\circledR}$, Sanofi, Paris, France), appears to be very promising, ${ }^{15}$ and is also effective against rhinosinusitis, which is seen in many patients with severe asthma, and is already approved as an effective treatment of severe atopic dermatitis. This is promising for patients with asthma who also have concomitant allergic diseases.

Anti-IL-17 treatment is more appropriate for treating non-eosinophilic or neutrophilic asthma because there is evidence that $\mathrm{IL}-17$ is an important driving mechanism for neutrophilic inflammation in asthma. Unfortunately, a clinical trial investigating an IL-17 receptor blocking antibody, which had shown effectiveness in psoriasis and inflammatory bowel disease, did not reach its endpoints but this may be due to the fact that the patients had not been selected for neutrophilic inflammation so any benefit would have been missed. ${ }^{16}$

\begin{abstract}
A thymic stromal lymphopoietin (TSLP) blocking antibody, tezepelumab, also looks very promising. ${ }^{17}$ This acts upstream of IL-5 and IL-13 and has demonstrated reduced exacerbation, improved lung function and improved symptoms. Its benefit appears to be independent of the blood eosinophil count, so it may treat a broader spectrum of asthma, and there is evidence that it targets the IL-5, IL-4 and IL-13 pathways. This may be an even more effective approach in the future for treating patients with severe asthma. $\square$
\end{abstract}

1. To T, Simatovic J, Zhu J, et al. Asthma deaths in a large provincial health system. A 10-year population-based study. Ann Am Thorac Soc. 2014:11:1210-7.

2. Global Initiative for Asthma (GINA). 2018 GINA Report, Global Strategy for Asthma Management and Prevention. Available Strategy for Asthma Management and Prevention. Available
at: https://ginasthma.org/2018-gina-report-global-strategy-forasthma-management-and-prevention/ (accessed 16 July 2018)

3. Partridge MR, van der Molen T, Myrseth SE, et al. Attitudes and actions of asthma patients on regular maintenance therapy: the INSPIRE study. BMC Pulm Med. 2006;6:13.

4. Custovic A, Johnston SL, Pavord I et al. EAACI position statement on asthma exacerbations and severe asthma. Allergy. 2013;68:1520-31.

5. Chung KF, Wenzel SE, Brozek JL, et al. International ERS/ATS guidelines on definition, evaluation and treatment of severe asthma. Eur Respir J. 2014;43:343-73.

6. O'Byrne PM, Jenkins C, Bateman ED. The paradoxes of asthma management: time for a new approach? Eur Respir $\rfloor$ 2017;50:1701103.
7. O'Byrne PM, FitzGerald JM, Bateman ED, et al. Inhaled combined budesonide-formoterol as needed in mild asthma. N Engl J Med. 2018;378:1865-76.

8. Bateman ED, Reddel HK, O'Byrne PM, et al. As-needed budesonide-formoterol versus maintenance budesonide in mild asthma. N Engl J Med. 2018;378:1877-87. Flood-Page P, Swenson C, Faiferman I, et al. A study to evaluate safety and efficacy of mepolizumab in patients with moderate persistent asthma. Am J Respir Crit Care Med 2007;176:1062-71

10. Haldar $\mathrm{P}$, Brightling $\mathrm{CE}$, Hargadon B, et al. Mepolizumab and exacerbations of refractory eosinophilic asthma. N Eng/ J Med. 2009;360:973-84.

11. Nair P, Pizzichini MM, Kjarsgaard M, et al. Mepolizumab for prednisone-dependent asthma with sputum eosinophilia. $N$ Eng/ J Med. 2009;360:985-93

12. Pavord ID, Korn S, Howarth P, et al. Mepolizumab for severe eosinophilic asthma (DREAM): a multicentre, double-blind, placebo-controlled trial. Lancet. 2012;380:651-9.
13. Bleecker ER, FitzGerald JM, Chanez P, et al. Efficacy and safety of benralizumab for patients with severe asthma uncontrolled with high-dosage inhaled corticosteroids and long-acting $\beta_{2}$-agonists (SIROCCO): a randomised, multicentre, placebo$\beta_{2}$-agonists (SIROCCO): a randomised, multicentre, $\mathrm{p}$
controlled phase 3 trial. Lancet. 2016;388:2115-27.

14. Castro $M$, Zangrilli J, Wechsler ME, et al. Reslizumab for inadequately controlled asthma with elevated blood eosinophi counts: results from two multicentre, parallel, double-blind, randomised, placebo-controlled, phase 3 trials. Lancet Resp Med. 2015;3:355-66.

15. Castro M, Corren J, Pavord ID, et al. Dupilumab efficacy and safety in moderate-to-severe uncontrolled asthma. N Engl J Med. 2018;378:2486-96.

16. Busse WW, Holgate S, Kerwin E, et al. Randomized, double-blind, placebo-controlled study of brodalumab, a human anti-IL-17 receptor monoclonal antibody, in moderate to severe asthma Am I Respir Crit Care Med. 2013:188:1294-302.

17. Corren J. Parnes JR Wang L et al. Tezepelumab in adults with uncontrolled asthma. N Engl J Med. 2017;377:936-46. 